\title{
Prototype of a System for Acquisition, Transmission and Visualization of Hydrometeorological Data Based on Raspberry Pi
}

\author{
Manuel Felipe Rodríguez Pérez, Esp, Javier Soto Vargas, PhD, \\ Escuela Colombiana de Ingeniería Julio Garavito, Colombia, \\ manuel.rodriguez-p@mail-escuelaing.edu.co, javier.soto@escuelaing.edu.co
}

\begin{abstract}
This document describes the implementation of the prototype of an acquisition, transmission and visualization system for hydrometeorological data, based on a single board computer (Raspberry Pi 2 Model B).The system includes 8 analog inputs, 2 digital inputs and 1 serial input with SDI-12 protocol. The information obtained from the sensors is acquired and stored in a local database. The measured data is displayed on a touch screen by means of a graphical interface for the configuration of parameters, management of the database, visualization of images and analysis of hydrometeorological data. According to a periodic condition defined by the user, the information is transmitted wirelessly to Hydras3, the national visualization system used in Colombia by the "Instituto de Hidrología Meteorología y Estudios Ambientales (IDEAM)". This is specialized software used for experts to analysis in the area of hydrology and meteorology. Additionally, the system integrates a camera that allows the capture and storage of images used to record different types of atmospheric phenomena, clouds, and climate changes such as melting or hydrological variables such as river levels and lakes.
\end{abstract}

Keywords-- Data collector platform, embedded system, hydrometeorological sensors, raspberry Pi, SDI-12 protocol, weather station.

\section{INTRODUCCIÓN}

A través de la historia el estudio de las variables hidrometeorológicas ha sido de interés especial para la sociedad, ya que estas influyen directamente en todas las actividades humanas. El conocimiento de la climatología hace posible que se estudien a fondo problemáticas de tipo ambiental, buscando entender los diversos factores que aceleran fenómenos como el cambio climático y los desastres naturales, los cuales pueden llegar a afectar negativamente al medio ambiente, a la flora, a la fauna y en muchas ocasiones la vida de los seres humanos.

Con el fin de estandarizar las mediciones realizadas a las diferentes variables hidrometeorológicas, la Organización Meteorológica Mundial (OMM) [1], ha establecido el programa del Sistema Global de Observaciones (GOS), el cual comprende estaciones de observación en tierra, en el mar, en el aire y en el espacio exterior. De acuerdo con la guía de instrumentos y métodos de observación de la OMM [2], dentro de las observaciones de superficie están las estaciones meteorológicas, que son espacios dedicados a la medición de variables, las cuales deben estar dotadas de instrumentos debidamente calibrados, fiables y precisos, con el fin de

Digital Object Identifier (DOI): http://dx.doi.org/10.18687/LACCEI2018.1.1.458 ISBN: 978-0-9993443-1-6

ISSN: $2414-6390$ satisfacer las necesidades de las medidas hidrometeorológicas deseadas.

En Colombia, el campo de la meteorología e hidrología es de gran importancia debido a la riqueza natural que existe en todo el territorio nacional. En la mayoría de los casos, los estudios de las diferentes variables climatológicas en el país, se realizan de forma tradicional, utilizando instrumentos convencionales en los que una persona observa las mediciones y anota la información de forma manual, lo cual implica una alta incertidumbre en la forma de medición, además de retardos en el proceso de registro y análisis de la información.

En Colombia, el Instituto de Hidrología Meteorología y Estudios Ambientales (IDEAM), cuenta con una red de estaciones hidrometeorológicas conformada por 2621 puntos de observación activos, de las cuales 2270 corresponden a estaciones de tipo convencional (registro manual de información), 305 son estaciones automáticas con transmisión de datos y 46 son estaciones automáticas de almacenamiento "in situ" [3]. En la Fig. 1, se puede observar la distribución porcentual de la red de estaciones hidrometeorológicas del IDEAM.

\section{Red de Estaciones IDEAM (Colombia)}

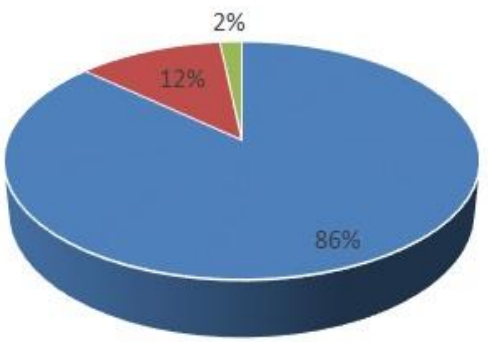

- Estaciones Convencionales
(Manuales)
- Estaciones Automaticas
con Transmision
- Estaciones Automaticas sin
Transmision

Fig. 1 Clasificación de Estaciones Hidrometeorológicas en Colombia.

Actualmente en Colombia se están remplazando los métodos de medición convencionales (manuales), por sistemas electrónicos automáticos, que permiten capturar información de sensores especializados y transmitirlos con una periodicidad horaria, sin embargo los dispositivos que actualmente realizan estas funciones son de fabricación extranjera lo cual implica que los costos sean altos, por lo cual la cantidad de puntos de medición son escasos con respecto a la extensión del país. El déficit de puntos de medición con transmisión en tiempo real hace que la información no sea

16 $^{\text {th }}$ LACCEI International Multi-Conference for Engineering, Education, and Technology: "Innovation in Education and Inclusion”, 19-21 July 2018, Lima, Peru. 
suficiente para realizar con precisión pronósticos climáticos y por ende prevenir por medio de alertas tempranas a poblaciones en riesgo de sufrir catástrofes naturales que se puedan llegar a presentar, también se reduce la calidad y veracidad de los estudios realizados en materia de energías renovables, salud, agricultura y calidad de agua, entre otros.

\section{ESTADO DEL ARTE}

Existen una serie de proyectos que han propuesto desarrollos similares para la medición de diferentes variables, utilizando registradores de datos comerciales (dataloggers) como los utilizados en [4] y [5], donde además se desarrolla software para la visualización de información de forma gráfica, conectándose a computadores mediante protocolos de comunicación cableados. Así mismo se utilizan tarjetas externas especializadas, como la que se utiliza en [6] donde adicionalmente se desarrollan sensores para el monitoreo de variables meteorológicas.

Otros desarrollos similares basan su funcionamiento en dispositivos microcontrolados, implementando estaciones meteorológicas de bajo costo como se explica en [7] y [8], en los cuales se integran sensores comerciales de la línea estudiantil para la medición de variables, y donde además se utilizan sistemas de comunicación inalámbrica, como los mostrados en [9] y [10] donde por medio de enlaces de radio y celular, se transmite la información adquirida a locaciones remotas con el fin de almacenarla y analizarla posteriormente.

También se encuentran proyectos donde utilizan computadores de placa reducida como la Raspberry Pi, y diversas herramientas de hardware y software libre para el desarrollo de interfaces gráficas, como en [11] y [12], donde se realiza la adquisición y procesamiento de información dentro del sistema embebido. Así mismo trabajos como los mostrados en [13] y [14], utilizan las diferentes interfaces de la Raspberry Pi para la conexión de sensores hidrometeorológicos especializados y la integración de módems para el acceso a internet transmitiendo la información a servidores web y aplicaciones móviles en smartphones. De igual forma el proyecto presentado en [15], utiliza la Raspberry Pi para el monitoreo de variables meteorológicas integrando un sistema de adquisición de imágenes mediante una cámara digital.

Finalmente en el mercado colombiano se encuentran diferentes plataformas colectoras de datos (DCP) de fabricación extranjera, como la NetDL1000 de OTT [16], la CR6 de Campbell Scientific[17] y la DA15K de Siap+Micros [18], las cuales son de uso profesional y cuentan con especificaciones técnicas de calidad superior, para la adquisición de datos de sensores hidrometeorológicos especializados ya sean de tipo análogo, digital y diferentes protocolos de comunicación como RS232, RS485 y SDI-12. Además este tipo de plataformas puede integrar sistemas de trasmisión de datos que puede ser de tipo radio, celular o satelital.

\section{COMPONENTES DEL PROTOTIPO DESARROLLADO}

El prototipo desarrollado consistió básicamente en el diseño e implementación de una plataforma colectora de datos (DCP), que permite adquirir, almacenar y transmitir vía internet, información de sensores hidrometeorológicos especializados, para lo cual se utilizó como sistema central de procesamiento la tarjeta Raspberry Pi 2 Model B [19], la cual es un computador de placa reducida que cuenta con características técnicas adecuadas para el desarrollo del prototipo, entre las que se encuentran una memoria RAM de $1 \mathrm{~GB}, 4$ puertos USB, 1 puerto Ethernet, 1 puerto dedicado para la conexión de cámara (CSI), y 1 puerto especial para conectar una pantalla táctil (DSI). Adicionalmente cuenta con un conector de 40 pines que permiten la interacción con otros dispositivos de lógica digital, cada uno de estos tiene una función específica, ya sea para alimentación (3.3V, 5V, GND), protocolos de comunicación (SPI, I2C, UART), o como puertos digitales configurables de entrada/salida a 3.3V (pines GPIO - General Purpose Input Output). En la Fig. 2, se puede observar la placa Raspberry Pi 2 Model B junto con la distribución y uso de puertos e interfaces utilizados en el desarrollo del prototipo.

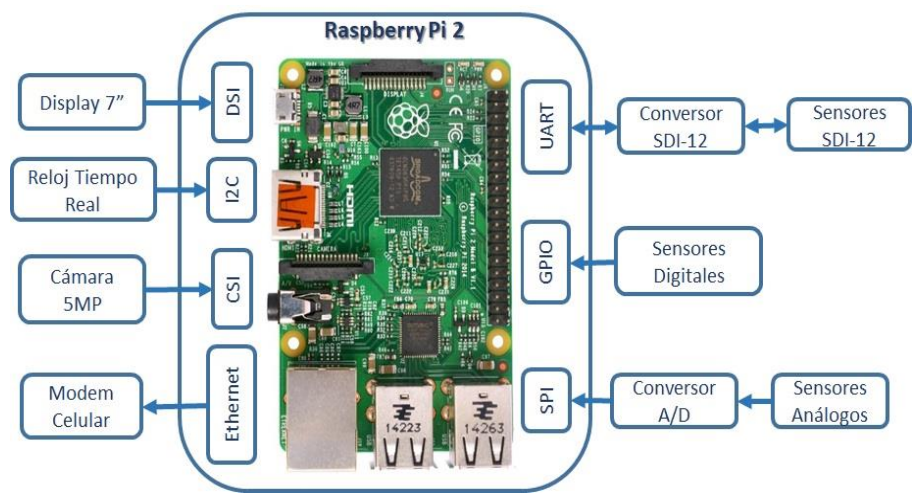

Fig. 2. Distribución de Puertos e Interfaces en la tarjeta Raspberry Pi

Basados en la revisión del estado del arte descrito en la sección II, en la verificación de tecnologías existentes en la actualidad, y en las especificaciones técnicas de los sensores electrónicos más utilizados en el campo de la meteorología, se decidió implementar el prototipo de una plataforma colectora de datos que cuente con ocho (8) entradas de tipo análogo en el rango de 0-5 V, dos (2) entradas digitales de pulsos, y una (1) entrada serial con protocolo SDI-12. Así mismo se decide integrar una cámara digital para la captura de imágenes fotográficas. A continuación se describe cada una de las entradas utilizadas para la lectura de sensores.

\section{A. Entradas Análogas}

Debido a que la tarjeta Raspberry Pi no cuenta con entradas para lectura de señales análogas, y con el propósito de integrar sensores de este tipo, se utilizó el conversor análogo digital MCP3008, el cual es un dispositivo que cuenta 
con 8 canales de entrada a una resolución de 10 bits y una interfaz serial SPI (Serial Peripheral Interface), que lo hace compatible con otros dispositivos que manejen el mismo estándar, y en este caso particular compatible con la Raspberry Pi utilizada. Se decide utilizar este dispositivo ya que de acuerdo con documentos técnicos de la OMM [20] [21], las plataformas colectoras de datos normalmente cuentan con un número de canales análogos comprendido entre 4 y 32, los cuales son particularmente importantes puesto que la mayoría de transductores meteorológicos utilizados para el sensado de variables como temperatura, humedad y presión, entregan una señal de voltaje directo, proporcional a la medición.

Con el fin de realizar la conexión entre la tarjeta principal y el conversor análogo digital, se decide utilizar un conversor de niveles lógicos basado en el transistor BSS138, ya que la Raspberry Pi maneja niveles lógicos de 0 a 3.3V, mientras que el MCP3008 utiliza para su comunicación niveles de 0 a $5 \mathrm{~V}$. Al utilizar el conversor de niveles lógicos se garantiza el correcto funcionamiento y la protección para los dos dispositivos. En la Fig. 3 se observa un diagrama de bloques que ilustra la conexión de los sensores análogos a la tarjeta Raspberry Pi.

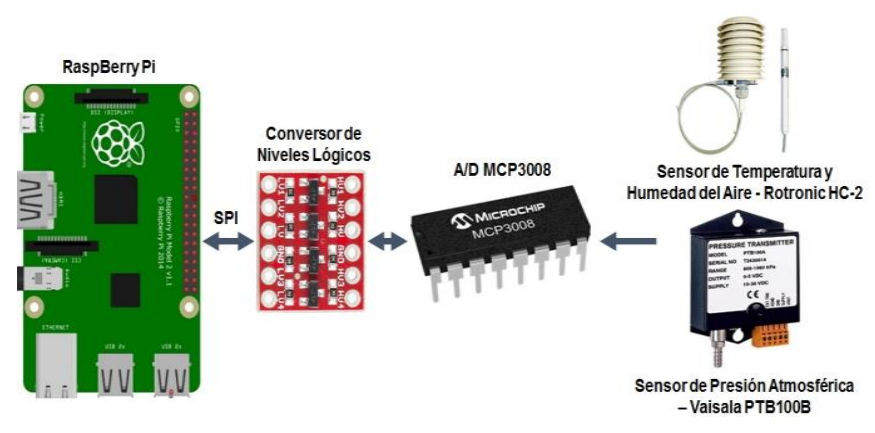

Fig. 3. Diagrama de Bloques Entradas Análogas.

\section{B. Entradas Digitales}

Los pines GPIO de la Raspberry Pi son configurables como entrada o salida digital a máximo $3.3 \mathrm{~V}$, además tienen la posibilidad de activar por software resistencias internas de pull-up o pull-down de acuerdo a la necesidad. Para la lectura de sensores con salida digital, cuya señal de salida está asociada a determinada cantidad de pulsos digitales, como los sensores de precipitación tipo balancín (tipping bucket rain gauge), se configuran dos puertos GPIO como entrada digital, cada uno de ellos con una resistencia interna de pull-up para garantizar la lectura del nivel alto y evitar ruidos en la señal, así mismo se utiliza una resistencia externa de $330 \Omega$ en cada pin, como protección en la lectura del pulso en nivel bajo. En la Fig. 4, se muestra un diagrama que ilustra la conexión utilizada para las entradas digitales del prototipo y el sensor de precipitación tipo balancín que se utilizó para pruebas del prototipo.

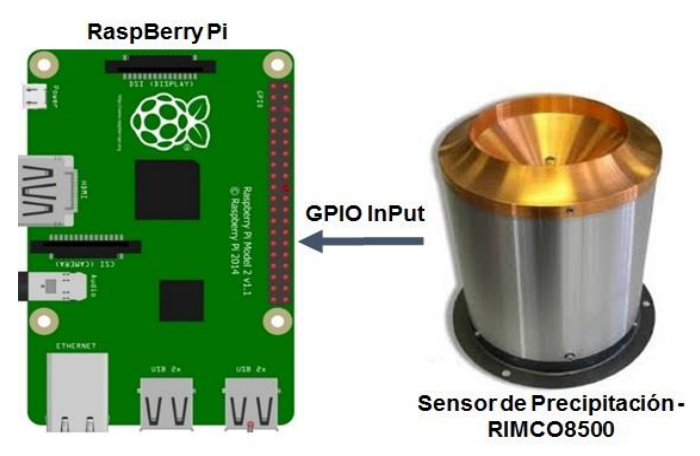

Fig. 4. Diagrama de Conexión Entradas Digitales por Pulsos

En el programa base desarrollado en Python3, para realizar la lectura de información de este tipo de sensores, se hace uso de las interrupciones de programa, para este caso se utiliza la interrupción por flanco de bajada (falling), con la cual, cuando se detecta un cambio de estado en el pin, generado por un pulso digital, el programa principal se detiene para dar paso a una función predefinida, la cual, basada en un factor ingresado por el usuario, según especificaciones técnicas del sensor (resolución), realiza la suma de la cantidad de pulsos detectados multiplicados por el valor de la resolución.

\section{Entradas Seriales SDI-12}

Con la finalidad de poder integrar instrumentos de medición de última tecnología, se hizo uso del protocolo serial SDI-12 (Serial Data Interface - 1200 Baud) [22], el cual es un estándar de comunicación asíncrona, que fue desarrollado para sensores e instrumentos inteligentes usados generalmente en el monitoreo de información medioambiental. El protocolo SDI12 utiliza un sistema Maestro-Esclavo, en la cual una unidad central de procesamiento, actúa como maestro y los sensores actúan como esclavos, permitiendo conectar hasta 62 sensores en paralelo. Una de las principales ventajas de este protocolo, es que únicamente utiliza para su comunicación entre dispositivos un bus de datos de tres líneas, una línea para los datos seriales, una línea de tierra y una línea de voltaje de alimentación.

Debido a que la tarjeta Raspberry Pi ejecuta varios procesos simultáneos para el funcionamiento adecuado del sistema operativo, para la lectura de sensores SDI-12 es preferible utilizar un sistema microcontrolado independiente, que se encargue del intercambio de comandos realizando la lectura del dato y entregándolo directamente a la tarjeta Raspberry Pi. Por consiguiente, se utilizó la placa Arduino Nano, la cual es una plataforma electrónica abierta para la creación de prototipos basada en software y hardware libres, y que con la ayuda de la librería especializada Arduino-SDI-12 realiza la sincronización de tiempos y el intercambio de comandos, conectando los sensores con salida SDI-12 directamente a los pines digitales de la placa Arduino sin

\footnotetext{
${ }^{1}$ Liberia Aurduino-SDI-12 originalmente escrita por Kevin Smith disponible en https://github.com/StroudCenter/Arduino-SDI-12[23]
}

$16^{\text {th }}$ LACCEI International Multi-Conference for Engineering, Education, and Technology: "Innovation in Education and Inclusion”, 19-21 July 2018, Lima, Peru. 
depender de ningún elemento externo. Adicionalmente, este dispositivo es de bajo costo, tamaño pequeño y permite la conexión física a la Raspberry Pi por medio de un cable USB, con el que se efectúa la alimentación y la comunicación por medio del protocolo serial. En la Fig. 5, se puede observar el diagrama de bloques que ilustra la conexión entre la tarjeta Raspberry Pi, la plataforma Arduino Nano y el sensor de vientos SDI-12 que se utilizó para pruebas del prototipo.

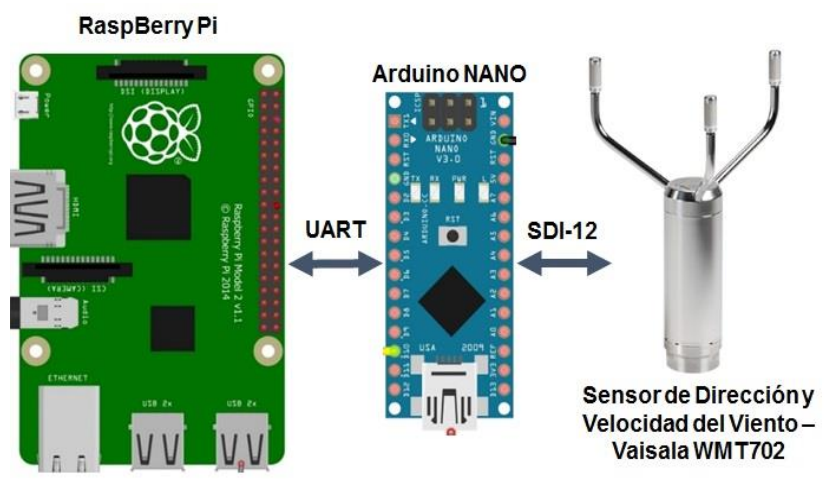

Fig. 5. Diagrama de bloques Entrada Serial SDI-12

\section{ARQuitectura, ProceSAMIENTO Y ViSUALIZACIÓN DE DATOS HIDROMETEOROLÓGICOS}

Para la adquisición y procesamiento de datos, se instaló y desarrolló el software necesario para el funcionamiento del prototipo. Inicialmente se realizó la instalación y configuración del motor de base de datos MySql, en el que se realiza el almacenamiento y organización de información mediante tablas de datos ordenadas.

Posteriormente se desarrolló una aplicación base utilizando Python3, la cual basada en la configuración establecida, utiliza los pines de propósito general de la Raspberry Pi para interactuar con dispositivos externos, encargándose de funciones como la lectura de datos de los sensores, su respectivo procesamiento y transformación en variables hidrometeorológicas, así como también la escritura de dicha información en las tablas de la base de datos. Esta aplicación en Python3 se encarga también de la creación del archivo de texto para el envío de datos y la conexión con el servidor remoto. Además es la responsable de la adquisición y almacenamiento de imágenes fotográficas.

Adicionalmente utilizando el entorno de desarrollo integrado MonoDevelop y su compilador de C\#, se desarrolló una interfaz gráfica, con el propósito de brindar al usuario un entorno amigable de configuración y visualización de cada una de las variables a medir, generando un archivo de texto en formato XML, en el que la aplicación de Python3 se basa para iniciar su funcionamiento. Esta interfaz muestra los valores adquiridos por los sensores, visualizándolos de forma tabular y de forma gráfica, de la misma manera admite configuración de la captura de imágenes, y la gestión sobre la base de datos, permitiendo realizar consultas, exportaciones y borrado de la información.

Todos los componentes de software son de uso libre para cualquier persona y se comunican entre sí para el funcionamiento adecuado del prototipo implementado. En la Fig. 6 se puede observar la estructura y componentes de software utilizados para el desarrollo del prototipo.

\section{A. Interfaz de Configuración de Parámetros y Visualización de Datos}

Debido a que es importante configurar y visualizar los datos de los sensores medidos de forma local, se utilizó una pantalla táctil de 7" conectada a la Raspberry Pi por medio del puerto DSI (Display Serial Interface) para mostrar una interfaz gráfica, la cual permite configurar de forma amigable los parámetros de los sensores y mostrar el comportamiento de cada una de las variables.

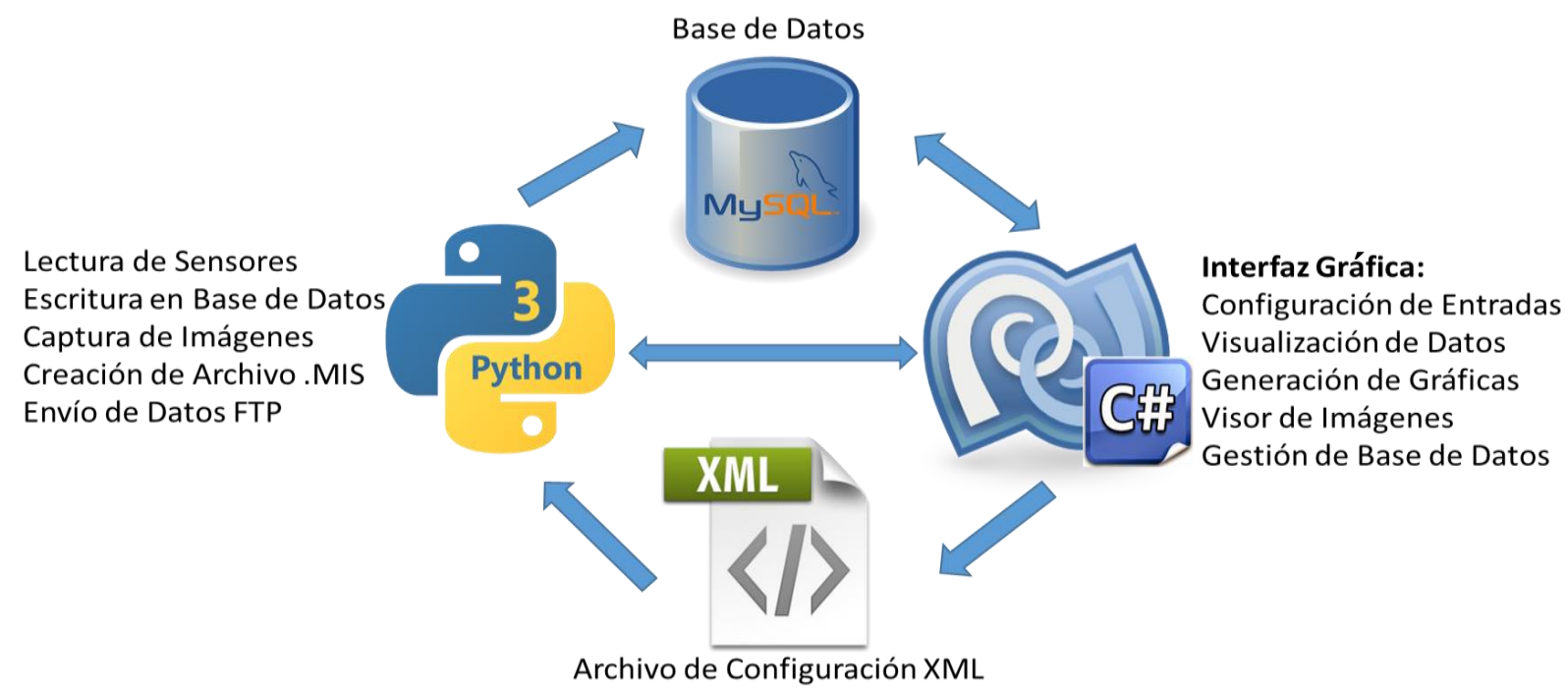

Fig. 6. Arquitectura de Software Implementada en el Prototipo

16 $^{\text {th }}$ LACCEI International Multi-Conference for Engineering, Education, and Technology: "Innovation in Education and Inclusion", 19-21 July 2018, Lima, Peru. 
Para el desarrollo de la aplicación gráfica se utilizó la plataforma de software MONO, por medio del entorno de desarrollo integrado (IDE) MonoDevelop, el cual cuenta con un compilador de $\mathrm{C \#}$, y una gran cantidad de librerías útiles para la conexión a base de datos MySQL y diferentes tipos de componentes como GTK\#, que es la herramienta especializada para entornos de aplicaciones gráficas, la cual contiene los objetos y funciones para crear instrumentos como ventanas, botones, menús, etiquetas, pestañas, etc.

La interfaz gráfica de visualización local está compuesta por pestañas o paneles con los que el usuario interactúa, permitiendo diferentes opciones para la configuración, visualización, almacenamiento y gestión de la información obtenida por los sensores hidrometeorológicos. A continuación, se describe cada uno de los paneles que componen la interfaz gráfica.

1) Panel de Configuración: En este panel, el usuario ingresa todos los datos necesarios para el funcionamiento adecuado de la adquisición y procesamiento de las variables, ingresando información asociada a la identificación, muestreo y parámetros de medición de acuerdo al tipo y características de los sensores, ya sean de tipo análogo, digital o serial con protocolo SDI-12. En la Fig. 7, se muestra una captura de pantalla del panel de configuración.

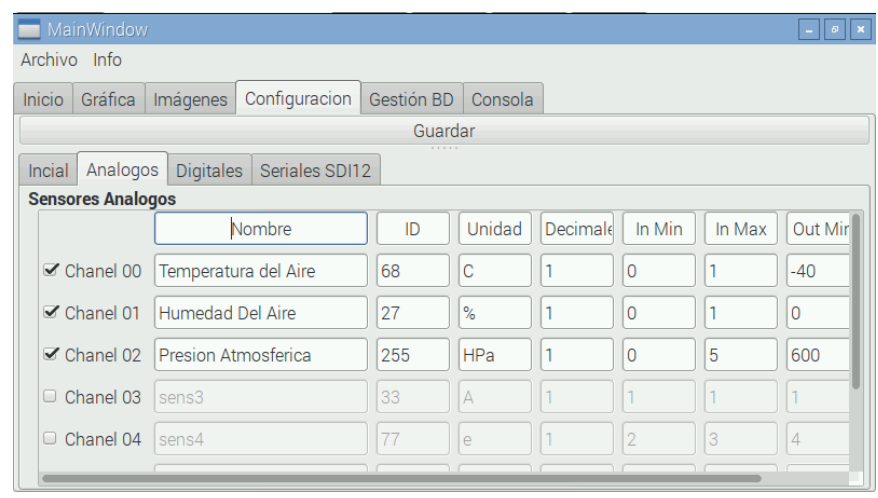

Fig. 7. Panel de Configuración

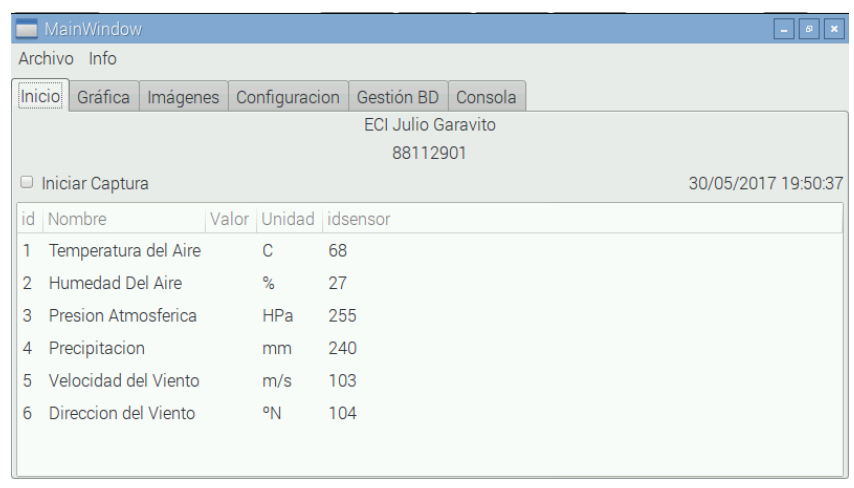

Fig. 8. Panel de Inicio
2) Panel de Inicio: Este panel es el espacio para visualizar en tiempo real los datos adquiridos por los sensores, estas variables se actualizan de acuerdo al tiempo de visualización, a los nombres, unidad e identificadores (id) establecidos previamente en el panel de configuración. En la Fig. 8, se presenta una captura de pantalla del panel de inicio.

3) Panel de Visualización Gráfica de Variables: Este panel está diseñado para realizar la visualización y análisis del comportamiento de las variables previamente adquiridas con respecto al tiempo, los datos son obtenidos del almacenamiento en la base de datos y sirven para revisar el comportamiento de una variable, o para diagnosticar el funcionamiento de los sensores hidrometeorológicos. La presentación de la información se realiza de forma gráfica y de forma tabular, permitiendo seleccionar cualquiera de las diferentes variables que están configuradas dentro de los parámetros de la estación. En la Fig. 9, se observa una captura de pantalla del panel de visualización gráfica de variables.

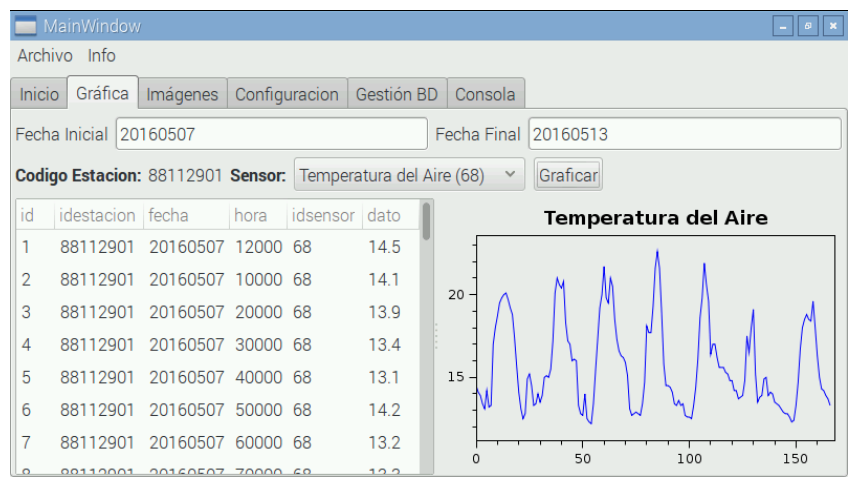

Fig. 9. Panel de Visualización Gráfica de Variables

4) Panel de Gestión de Base de Datos: En este panel se pueden realizar consultas asociadas a los parámetros almacenados en la base de datos por medio de comandos básicos SQL. Dentro del panel de gestión existe la posibilidad de realizar la exportación de la información consultada a un archivo de texto plano separado por punto y coma ",", el cual tendrá una extensión ".CSV", este tipo de archivos se puede abrir fácilmente con programas ofimáticos para manejo de hojas de cálculo como Excel o LibreOfficeCalc. Otra funcionalidad con la que cuenta este panel es la posibilidad de eliminar registros almacenados en la base de datos. En la Fig. 10 , se observa una captura de pantalla del panel de gestión de base de datos.

16 $^{\text {th }}$ LACCEI International Multi-Conference for Engineering, Education, and Technology: "Innovation in Education and Inclusion", 19-21 July 2018, Lima, Peru. 


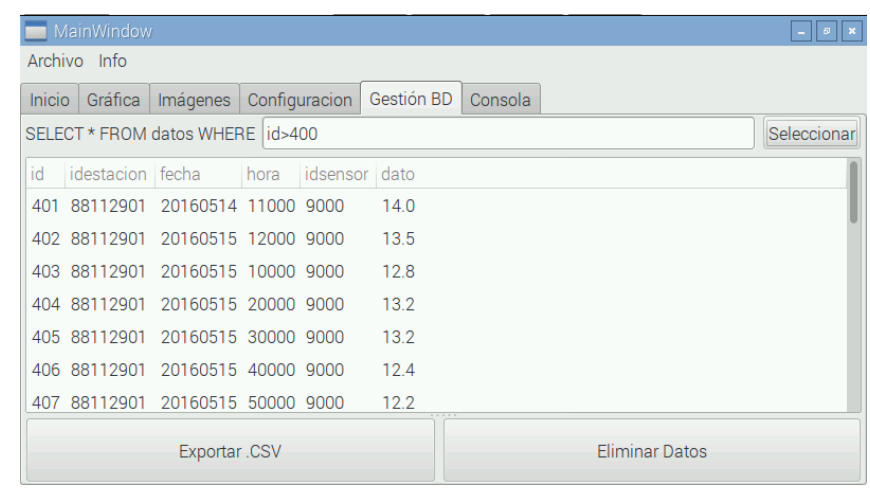

Fig. 10. Panel de Gestión de Base de Datos

\section{B. Adquisición de Imágenes}

El propósito de la adquisición de imágenes, es realizar la captura de fotografías del entorno donde se encuentre ubicado el prototipo, de acuerdo a una condición periódica preestablecida por el usuario, guardando dichas imágenes dentro del espacio de almacenamiento no volátil de la Raspberry Pi, brindando la posibilidad de registrar gráficamente observaciones de fenómenos atmosféricos como meteoros ${ }^{2}$ y nubes, cambios ambientales como el deshielo o variables hidrológicas como niveles de ríos y embalses, para así tener otra fuente de información con el fin de establecer criterios de estados de alerta y posibles soluciones.

Para la adquisición de imágenes se utiliza una cámara de 5 Mpx, conectada a la Raspberry Pi mediante el puerto CSI (Camera Serial Interface), la cual es compatible con el sistema operativo y cuenta con una librería especializada para realizar aplicaciones en Python3. En la Fig. 11, se observa la interfaz de captura de imágenes implementada para el prototipo.

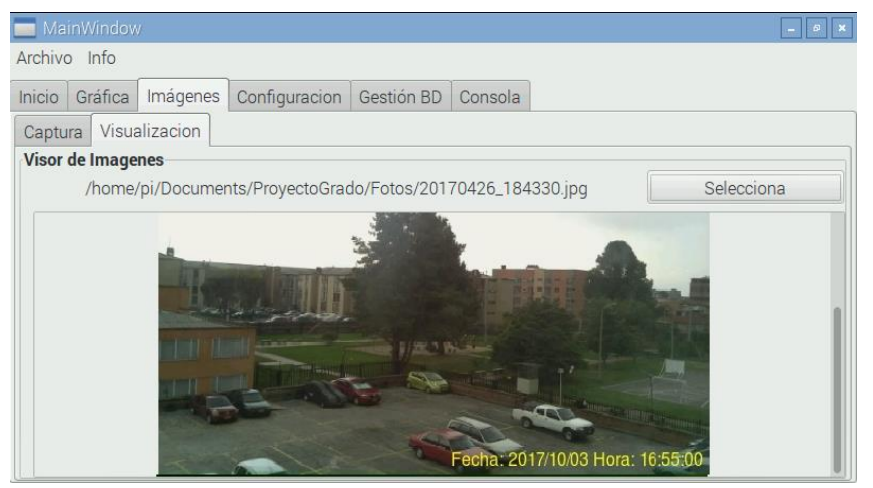

Fig. 11. Interfaz Gráfica de Captura de Imágenes

\footnotetext{
2 De acuerdo con la OMM un meteoro es "un fenómeno observado en la atmósfera o sobre la superficie de la tierra, que consiste en una suspensión, una precipitación, o un depósito de partículas líquidas, acuosas o no, o de partículas sólidas, o un fenómeno de la naturaleza de manifestación óptica o eléctrica." [24] por ejemplo: neblina, tempestad de arena, arco iris, halo solar y relámpagos, entre otros.
}

\section{SISTEMA DE COMUNICACIÓN INALÁMBRICA Y SOFTWARE DE VISUALIZACIÓN REMOTO}

Dada la necesidad de poder centralizar la información de los datos registrados por los diferentes sensores hidrometeorológicos, se decide utilizar un sistema de transmisión inalámbrico mediante el servicio de comunicación GPRS (General Packet Radio Service), para lo cual se utilizó el módem celular MOXA OneCell G3151, junto con una tarjeta SIM y un plan de datos celular convencional permitiendo el acceso a internet de la tarjeta Raspberry Pi por medio de una conexión Ethernet.

La aplicación desarrollada en Python3 se encarga de consultar los datos almacenados en la base de datos, así como de ordenarlos en un archivo de texto plano con una estructura predeterminada, el cual es enviado vía internet hacia un servidor remoto mediante el protocolo FTP (File Transfer Protocol). Con el fin de visualizar esta información, se realizó la integración con el software de visualización de estaciones meteorológicas automáticas del IDEAM, conocido como Hydras3, el cual es un programa licenciado y desarrollado por la empresa alemana OTT Hydrmet, que cuenta con una interfaz web para el acceso a la información de forma remota desde cualquier parte del mundo con conexión a internet.

Hydras3 interpreta la información enviada en el archivo de texto plano, permitiendo la visualización de datos en forma gráfica y en forma tabular, la cual sirve para realizar el análisis e interpretación de los valores de cada una de las variables hidrometeorológicas, y que es utilizado para realizar análisis numéricos, presentación de informes y configuración de alertas de toda la red de estaciones. En la Fig. 12, se puede observar el esquema de comunicación inalámbrica utilizado en el prototipo.

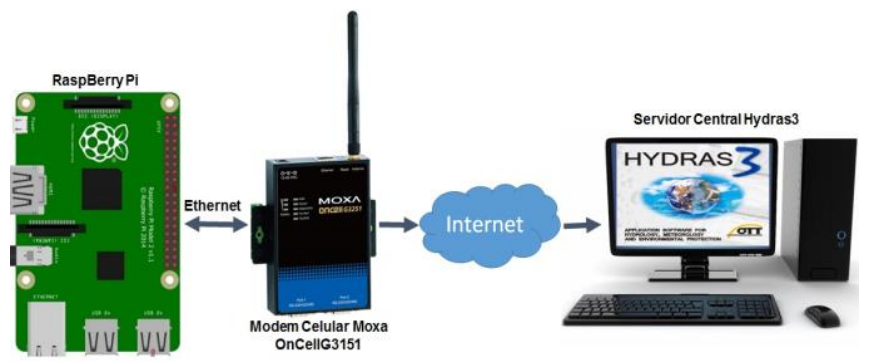

Fig. 12. Sistema de Comunicación Inalámbrica

Se realizó la integración de los datos capturados por el prototipo al software Hydras3, verificando la estructura del archivo de texto enviado vía internet y la correcta recepción en el servidor FTP del IDEAM, con lo cual se realizaron pruebas de visualización de datos en el software durante un periodo de tiempo superior a 48 horas de funcionamiento continuo, con periodos de muestreo de 2 minutos, registrando valores de sensores de tipo análogo de temperatura del aire, humedad del aire, y presión atmosférica, un sensor digital de precipitación por pulsos, y un sensor serial con protocolo SDI-12 de

$16^{\text {th }}$ LACCEI International Multi-Conference for Engineering, Education, and Technology: "Innovation in Education and 
dirección y velocidad del viento, obteniendo 8687 registros transmitidos y visualizados de forma adecuada en Hydras3. En la Fig. 13, se muestra de forma gráfica los datos obtenidos para la variable análoga de humedad del aire durante un periodo de tiempo de 3 días en un ambiente cerrado; en la parte superior se puede observar los datos mostrados en la interfaz gráfica del prototipo, mientras que en la parte inferior se muestran los datos que fueron recibidos en el software Hydras3. De esta gráfica se puede inferir el adecuado comportamiento de la variable capturada de humedad del aire, además de comprobar la transmisión exitosa de la información.

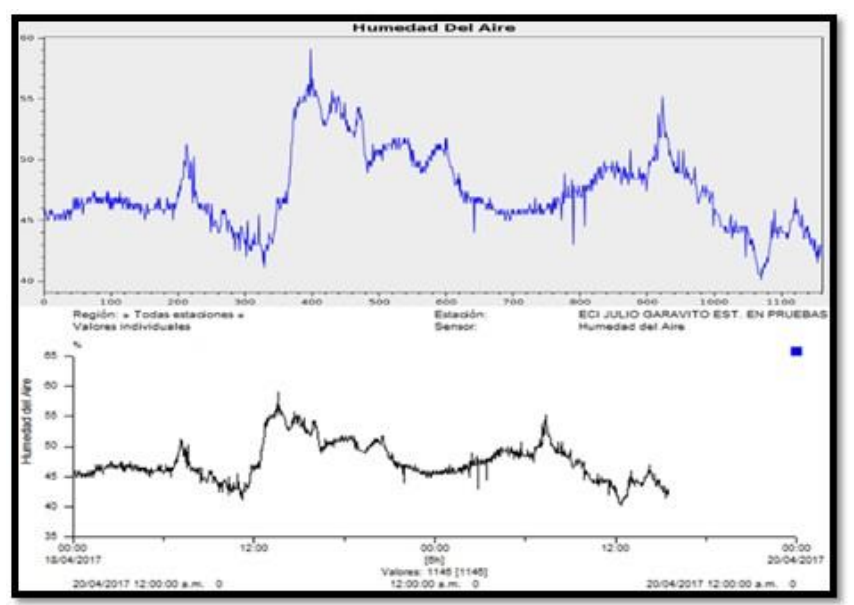

Fig. 13. Comparación de datos almacenados en el prototipo contra los datos recibidos en Hydras3

\section{RESULTADOS Y CONCLUSIONES}

Se diseñó e implementó el prototipo de una plataforma colectora de datos (DCP), basado en el computador de placa reducida Raspberry Pi 2 Modelo B, el cual es capaz de adquirir, almacenar, visualizar y transmitir datos hidrometeorológicos de sensores especializados. El almacenamiento de la información se hace con una base de datos relacional desarrollada en MySQL. El prototipo realiza el envío periódico de las variables registradas al software de visualización nacional del IDEAM Hydras3. Adicionalmente integra un sistema de registro fotográfico que permite capturar y almacenar imágenes en la memoria para su posterior análisis.

El sistema es capaz de realizar la captura de información de hasta 8 sensores de tipo análogo entre 0 y $5 \mathrm{~V}, 2$ sensores digitales con salidas en pulsos, y 1 entrada para sensores seriales con protocolo SDI-12. Cada una de las entradas para los sensores se puede configurar de forma personalizada por medio de una interfaz gráfica, ingresando los valores de los parámetros correspondientes a cada tipo de sensor. Las características del prototipo desarrollado son comparables con las características de las plataformas colectoras de datos comerciales más utilizadas actualmente en el mercado.
Para el desarrollo del prototipo se utilizó el sistema operativo, oficial de Rapsberry Pi conocido como Raspbian Jessie, el cual es una distribución open-source adaptada de Linux Debian, y por medio del cual se realizó todo el desarrollo de software implementado en la interfaz gráfica, utilizando herramientas y lenguajes de programación de uso libre como Python, MONO, MonoDevelop y C\#.

Se integró un sistema de captura de imágenes a la plataforma colectora de datos, convirtiéndose en un componente de innovación para el apoyo a las observaciones hidrometeorológicas, ya que en la actualidad, ninguna de las estaciones meteorológicas del IDEAM, cuenta con este tipo de sistemas fotográfico, lo cual puede servir como un registro alternativo para medición de variables como deshielo o nivel de ríos y embales, así como también el monitoreo de diversos fenómenos atmosféricos.

El prototipo cuenta con un conversor análogo digital con resolución de 10 bits, con lo cual se pueden realizar mediciones de varios sensores análogos que se encuentran en el mercado, sin embargo de acuerdo a los resultados obtenidos en las pruebas de funcionamiento, como trabajo futuro y con el fin de mejorar el funcionamiento del prototipo, se recomienda utilizar un conversor análogo digital de mayor resolución y exactitud, para realizar capturas de sensores que manejan salidas de voltaje en el orden de los micro voltios.

Se fabricó una placa base con el circuito implementado para la lectura de señales análogas, digitales y seriales con protocolo SDI-12, se utilizó un gabinete con protección NEMA $4 \mathrm{X}^{3}$ para la protección de los componentes electrónicos y una estructura física para la ubicación de los sensores de prueba, con lo cual se pudo verificar satisfactoriamente el funcionamiento del diseño planteado. En la Fig. 14, se puede observar el prototipo desarrollado junto con la estructura física utilizada para su montaje.
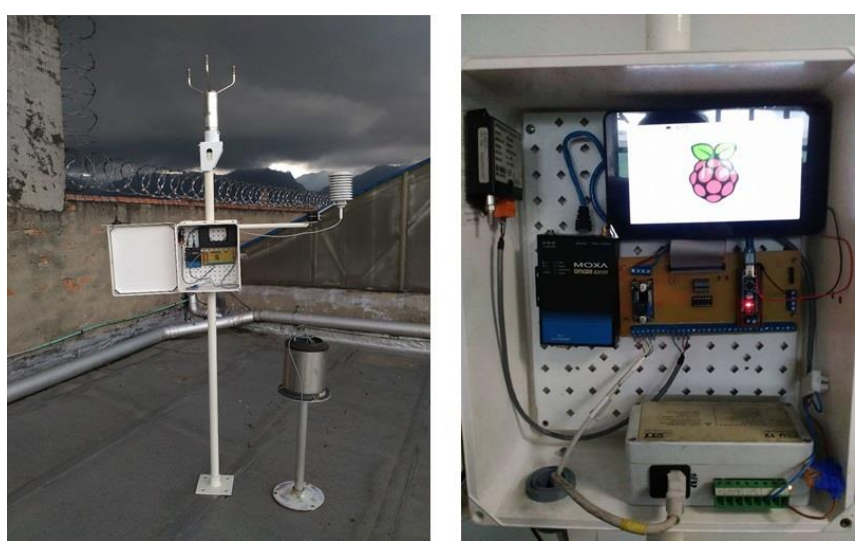

Fig. 14. Prototipo Desarrollado

\footnotetext{
${ }^{3}$ NEMA 4X es el estándar establecido por la Asociación Nacional de Fabricantes Eléctricos para equipos de instalación en exterior o trabajo en ambientes hostiles con un grado de protección contra: Polvo llevado por el viento y lluvia, Salpicadura de agua, Agua directa de la manguera, Daño por la formación exterior de hielo, Resistente a la corrosión.
} 


\section{AGRADECIMIENTOS}

Los autores agradecen a la Escuela Colombiana de Ingeniería Julio Garavito y al programa de Maestría en Ingeniería Electrónica por su respaldo y acompañamiento durante el proceso de formación académica, así mismo agradecen al Instituto de Hidrología Meteorología y Estudios Ambientales (IDEAM) por su ayuda y colaboración en el préstamo de sensores y elementos de prueba.

\section{REFERENCIAS}

[1] (OMM) Organización Meteorológica Mundial, «OMM en pocas palabras/OMM». [En línea]. Disponible en: https://www.wmo.int/pages/about/index_es.html. [Accedido: 24-mar2016].

[2] World Meteorological Organization (WMO), Guide to Meteorological Instruments and Methods of Observation. 2008.

[3] IDEAM, «Catalogo Nacional de Estaciones IDEAM». Actualización de Enero-2016.

[4] Q. Xuewu y C. Tijing, «Design and implementation of data acquisition system based on Agilent 3458A», en 2013 IEEE 11th International Conference on Electronic Measurement Instruments (ICEMI), 2013, vol. 1, pp. 28-31.

[5] M. Branzila, F. Mariut, y D. Petrisor, «Virtual instrument developed for adcon weather station», en 2012 International Conference and Exposition on Electrical and Power Engineering (EPE), 2012, pp. 853856.

[6] D. G. C. Niño, G. A. M. Vargas, y M. F. R. Perez, «Monitoring system of a weather station via IP», en 2010 IEEE ANDESCON, 2010, pp. 1-6.

[7] A. Shaout, Y. Li, M. Zhou, y S. Awad, «Low cost embedded weather station with intelligent system», en Computer Engineering Conference (ICENCO), 2014 10th International, 2014, pp. 100-106.

[8] O. Krejcar, «Low cost weather station with remote control», en 2012 IEEE 10th International Symposium on Applied Machine Intelligence and Informatics (SAMI), 2012, pp. 463-468

[9] R. Lajara, J. Alberola, J. Pelegri, T. Sogorb, y J. V. Llario, «Ultra Low Power Wireless Weather Station», en International Conference on Sensor Technologies and Applications, 2007. SensorComm 2007, 2007, pp. 469-474.

[10] M. Popa y C. Iapa, «Embedded weather station with remote wireless control», en Telecommunications Forum (TELFOR), 2011 19th, 2011, pp. 297-300.

[11] R. C. Brito, F. Favarim, G. Calin, y E. Todt, «Development of a low cost weather station using free hardware and software», en 2017 Latin American Robotics Symposium (LARS) and 2017 Brazilian Symposium on Robotics (SBR), 2017, pp. 1-6.

[12] R. Shete y S. Agrawal, «IoT based urban climate monitoring using Raspberry Pi», en Communication and Signal Processing (ICCSP), 2016 International Conference on, 2016, pp. 2008-2012.

[13] T. Savić y M. Radonjić, «One approach to weather station design based on Raspberry Pi platform», en Telecommunications Forum Telfor (TELFOR), 2015 23rd, 2015, pp. 623-626.

[14] P. Xie et al., «Sensing wind for environmental and energy applications», en 25th IET Irish Signals Systems Conference 2014 and 2014 China-Ireland International Conference on Information and Communications Technologies (ISSC 2014/CIICT 2014), 2014, pp. 6469.

[15] S. Jindarat y P. Wuttidittachotti, «Smart farm monitoring using Raspberry Pi and Arduino», en 2015 International Conference on Computer, Communications, and Control Technology (I4CT), 2015, pp. 284-288.

[16] «OTT netDL Data Logger-OTT Hydromet USA». [En línea]. Disponible en: http://www.ott.com/en-us/products/data-logging-andtelemetry-4/ott-netdl-data-logger-972/. [Accedido: 15-mar-2018].
[17] «CR6 - Measurement and Control Datalogger». [En línea]. Disponible en: https://www.campbellsci.com/cr6. [Accedido: 15-mar-2018].

[18] «Sistemas de Adquisición de Datos - Siap+Micros». [En línea]. Disponible en: http://www.siapmicros.com/es/prodotti/sistemas-deadquisicion-de-datos/. [Accedido: 15-mar-2018].

[19] «Raspberry Pi 2 Model B», Raspberry Pi. [En línea]. Disponible en: https://www.raspberrypi.org/products/raspberry-pi-2-model-b/. [Accedido: 06-may-2016].

[20] P. Battista, (WMO) World Meteorological Organization, (IATA) Institute of Agrometeorology and Environmental Analysis for Agriculture, y (CNR) Regional Meteorological Training Center, Manual on instrumentation and operations for automatic weather stations for agrometeorological application. WMO, 2000.

[21] WMO y World Meteorological Organization, Eds., Automatic weather stations. Geneva: WMO, 1963.

[22] SDI-12 Support Group, «SDI-12 A Serial-Digital Interface Standard for Microprocessor-Based Sensors Version 1.4». 10-ago-2016.

[23] «EnviroDIY/Arduino-SDI-12», GitHub. [En línea]. Disponible en: https://github.com/EnviroDIY/Arduino-SDI-12. [Accedido: 23-abr2017].

[24] Organización Meteorológica Mundial, Atlas internacional de nubes. 1, Manual de observación de nubes y otros meteoros 1, Manual de observación de nubes y otros meteoros. Ginebra (Suiza): Organización Meteorológica Mundial, 1993.

$16^{\text {th }}$ LACCEI International Multi-Conference for Engineering, Education, and Technology: "Innovation in Education and Inclusion”, 19-21 July 2018, Lima, Peru. 